\title{
EDUCA \& AÇÃO: UMA METODOLOGIA ATIVA VOLTADA AO CURSO DE ENGENHARIA PARA SIMULAR A REALIDADE POR MEIO DO JOGO
}

Daniel Alves dos Santos - kdanielalvesw@ gmail.com

Instituto Federal de Educação, Ciência e Tecnologia do Tocantins, Campus Palmas AE 310 Sul, Avenida LO 05, s/n, Plano Diretor Sul

77.021-090 - Palmas - Tocantins

Gustavo Yuji Ito - gustavo.yuito@gmail.com

Instituto Federal de Educação, Ciência e Tecnologia do Tocantins, Campus Palmas AE 310 Sul, Avenida LO 05, s/n, Plano Diretor Sul

77.021-090 - Palmas - Tocantins

Pedro Ignácio Meneghetti Scheid - p.scheid22@gmail.com

Instituto Federal de Educação, Ciência e Tecnologia do Tocantins, Campus Palmas AE 310 Sul, Avenida LO 05, s/n, Plano Diretor Sul

77.021-090 - Palmas - Tocantins

Héllica Machado Barbosa - hellica_machado@hotmail.com

Instituto Federal de Educação, Ciência e Tecnologia do Tocantins, Campus Palmas AE 310 Sul, Avenida LO 05, s/n, Plano Diretor Sul

77.021-090 - Palmas - Tocantins

VirleyLemos de Souza-virley@ifto.edu.br

Instituto Federal de Educação, Ciência e Tecnologia do Tocantins, Campus Palmas AE 310 Sul, Avenida LO 05, s/n, Plano Diretor Sul

77.021-090 - Palmas - Tocantins

Resumo: Colocar o aluno como o protagonista do seu próprio ensino e extrapolar as barreiras da sala de aula convencional favorece seu aprendizado. Uma das formas de alcançar isso é adotar metodologias ativas no processo, já que interações positivas nas aulas contribuem também com o conhecimento do aluno. Partindo desse ponto, foi desenvolvida uma metodologia por meio da gamificação para ser usada na graduação. Para isso, foram aplicadas as estruturas do Octalysis e dos jogos sérios, para encontrar e adaptar o sistema de RPG que mais se enquadra na sala de aula: o FIASCO. Assim foi desenvolvido o Educa \& Ação, um sistema voltado a trabalhar competências muitas vezes não tratadas na faculdade, enquanto o graduando tem uma aula diferente, divertida e cooperativa. Foram realizados testes no curso de engenharia civil do IFTO Campus Palmas nas disciplinas de Planejamento e Gerenciamento de Obras, da matriz curricular do $8^{\circ}$ Período, e Gestão da Qualidade e Produtividade, do $10^{\circ}$ Período. Os resultados obtidos pelos feedbacks dos alunos foram positivos: 96,7\% vê no Educa \& Ação uma ferramenta que contribuí na sua graduação, 93,3\% jogaria novamente e $100 \%$ se divertiu enquanto jogava. Com uma nota de experiência geral de 8,2, o Educa \& Ação se mostra uma metodologia validada para ensino na engenharia civil. 
Palavras-chave: Ensino, metodologia ativa, RPG, engenharia civil.

\section{INTRODUÇÃO}

De acordo com Querino \& Borges (2002), a formação do engenheiro, atualmente, demanda a mobilização de diferentes áreas do conhecimento, uma vez que se espera um profissional capaz de propor soluções que sejam não apenas tecnicamente corretas, mas também com ambição para considerar os problemas em sua totalidade, em sua inserção numa cadeia de causas e efeitos de múltiplas dimensões. Dessa forma, tem-se o desafio de propor novas metodologias educacionais que permitam aos novos engenheiros aplicar não somente as competências e habilidades técnicas para esta profissão, como também a capacidade de reflexão frente às realidades socioeconômicas, culturais e políticas do contexto em que vivem.

Além disso, segundo o Instituto Euvaldo Lodi - IEL (2006), considerando o contexto do mercado da engenharia, constata-se que os engenheiros recém-formados possuem bom conhecimento técnico mas demonstram dificuldades no que se refere aos pontos relacionados às atividades como: iniciativa e ação empreendedora, capacidade de gestão, comunicação, liderança e trabalho em equipes multidisciplinares.

À vista disso, pesquisas e trabalhos vêm sendo propostos para melhorar o processo educativo e satisfazer as necessidades da sociedade contemporânea quanto ao profissional de engenharia. Querino \& Borges (2002) por exemplo, inferem a consideração do aluno como sujeito ativo no processo de produção do conhecimento e a ampliação da aprendizagem com a promoção de experiências que extrapolam o ambiente de sala de aula. O IEL (2006) também afirma que, para que os recém-formados estejam mais preparados para a realidade do mercado, o foco deve deixar de ser o ensino e passar a ser a aprendizagem, com a proposição de novos modelos de gestão e avaliação de cursos, aliado a busca por novos métodos e meios de ensino/aprendizagem.

A partir daí, os conceitos de metodologias ativas, modelagem, gamificação, entre outros, vem surgindo como alternativas promissoras para estabelecer um sistema de ensino com mais qualidade e eficiência. A UNESCO (2013), através do projeto Laboratorio Latinoamericano de Evaluación de la Calidad de la Educación (LLECE) vem realizando pesquisas para mensurar a qualidade da educação nos países latinos para orientar as políticas educacionais. No estudo TERCE, de 2013, foi avaliado que o clima socioemocional e as interações positivas na sala de aula têm uma alta associação com a aprendizagem. Nesse sentido, é necessária uma nova geração de estratégias de desenvolvimento profissional do professor, diretamente ligada à prática em sala de aula.

Diante disso, o presente artigo estuda o desenvolvimento e aplicação de um jogo em algumas disciplinas do curso de engenharia civil, estruturado a partir dos conceitos de gamificação, estrutura Octalysis e jogos sérios, inspirado nas mecânicas e nos elementos de um Role Playing Game (RPG). Este simula situações reais que um profissional de engenharia enfrenta, sendo capaz de melhorar a proatividade dos alunos e a interação social devido às características intrínsecas presentes em jogos, além da possibilidade de adquirir a experiência necessária na problemática proposta pelo professor para solucioná-la com sucesso no futuro no ofício da sua profissão. 


\section{PERCURSO METODOLÓGICO}

\subsection{A gamificação e a estrutura Octalysis}

De acordo com Chou (2019), a gamificação é o ato de adaptar os elementos divertidos dos jogos e aplicá-los no mundo real em atividades do ser humano, focando o design no ser humano. Grande parte dos sistemas se foca na produtividade e funções de cada um, esquecendo que as pessoas dentro do sistema têm inseguranças, sentimentos e razões que reduzem seu engajamento e efetividade.

A razão do termo gamificação é devido a indústria de jogos ter sido a pioneira no domínio do design feito para o ser humano e, como eles passaram décadas estudando como controlar a motivação e engajamento das pessoas, agora é a vez dos outros ramos e indústrias aprenderem com os jogos, segundo Chou (2019). A estrutura Octalysis surgiu dos seus estudos das relações humanas com os jogos. Dividida em oito aspectos chamados de Cores Drives, que são tipos de motivadores, melhores descritos na Figura 1 mostrada a seguir.

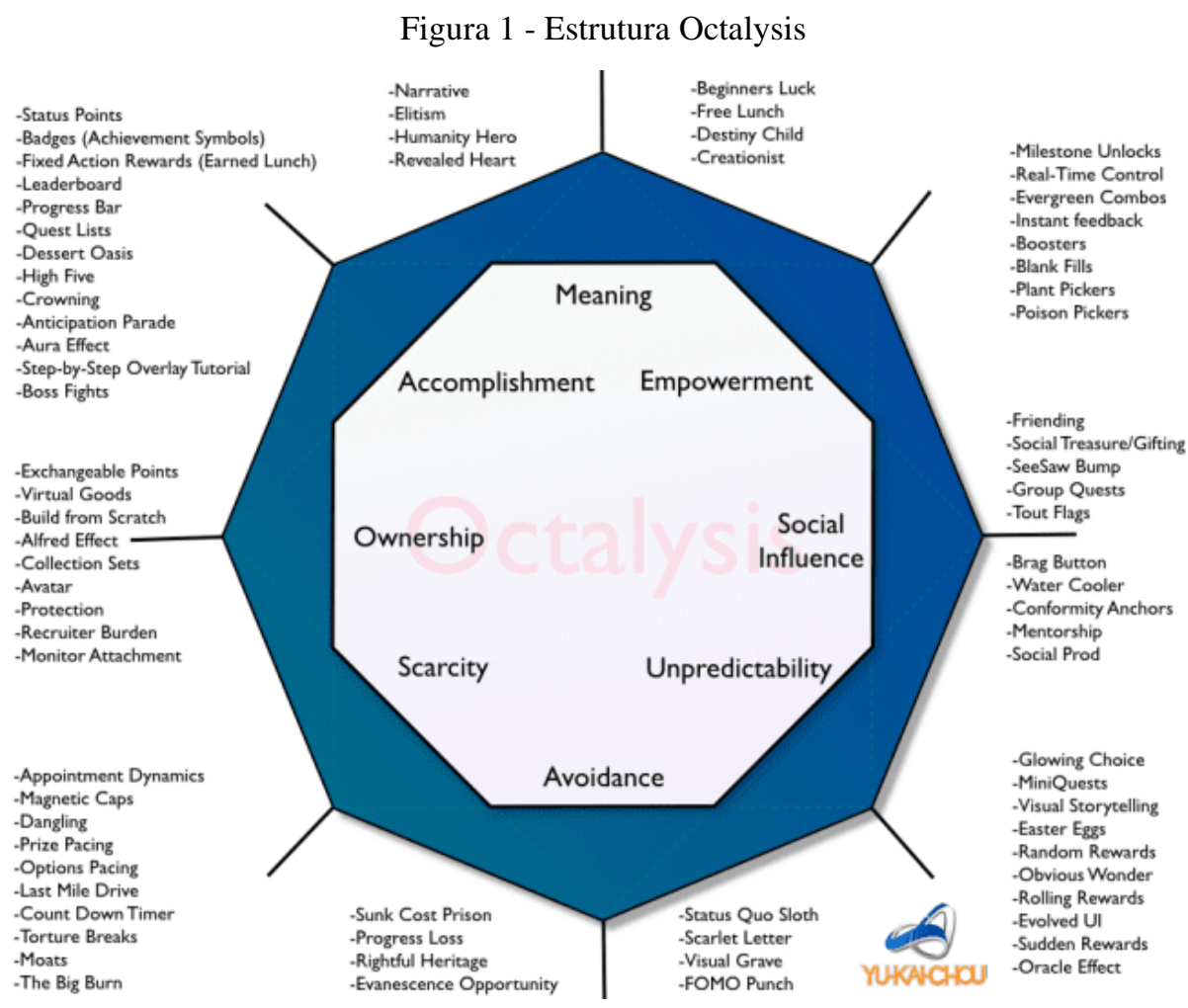

Fonte: Chou (2019)

Chou (2019) trabalha os Cores Drives de forma a evidenciar as características de competência, relacionamento e autonomia do ser humano dentro dos jogos, a seguir cada perspectiva é melhor descrita:

- Epic Meaning \& Calling - Sentido Épico e Chamada: O jogador se sente escolhido para fazer parte de algo maior, que o torna especial e o motiva. 
- Development \& Accomplishment - Desenvolvimento e Realização: Impulso do jogador de evoluir, desenvolver habilidades e enfrentar desafios, este último muito importante para aumentar a significância da superação e realização.

- Empowerment of Creativity \& Feedback - Capacidade de Criatividade e Feedback: O jogador é engajado pelo processo de criação, repetindo a atividade com combinações diferentes a cada tentativa, sendo que ao final de cada vez o mesmo veja o resultado de sua criatividade.

- Ownership \& Possession - Propriedade e Posse: O jogador é motivado pelo sentimento de possuir algo, querendo sempre mais e melhor. Opções de personalização das suas posses também são vistas nessa perspectiva.

- Social Influence \& Relatedness - Influência social e parentesco: Impulso do jogador buscar os elementos sociais como aceitação, companheirismo, competição, inclui ainda os impulsos sociais de se aproximar de certas pessoas e lugares nostálgicos ou especiais para nós.

- Scarcity \& Impatience - Escassez e impaciência: Motivação por querer algo que não se pode ter, o fato de não conseguir algo no momento a instiga a pensar naquilo.

- Unpredictability \& Curiosity - Imprevisibilidade e curiosidade: O jogador tem o desejo de descobrir o que vem a seguir, esse impulso também é excessivamente presente nos vícios em jogos de azar.

- Loss \& Avoidance - Perda e prevenção: Esse núcleo foca na prevenção do acontecimento de algo negativo ou a perda de atividades feitas anteriormente.

\subsection{Jogos Sérios}

De acordo com Rocha, Bittencourt \& Isotani (2015), "jogos sérios, em inglês serious games, são jogos utilizados com o propósito de ensino e aprendizagem ou treinamento e não apenas de entretenimento" (apud Aldrich, 2005; BNDES, 2014a) e contribuíram em diversas áreas da sociedade, como na saúde, turismo, defesa, dentre outras (apud Aldrich, 2005; Mattar, 2010; Novak, 2010).

Apesar disso, Rocha, Bittencourt \& Isotani (2015) afirmam que desenvolver esses jogos sérios é uma atividade complexa, devido à necessidade de integração de diversas áreas de conhecimento, do desenvolvedor de jogos, da área de aplicação do jogo, e de conhecimentos educacionais (apud Balci, 2012; EMS, 2006). A produção dos jogos sérios passa por sete principais desafios durante sua concepção, definidos por Rocha, Bittencourt \& Isotani (2015), que estão melhor detalhados conforme a Figura 2. 
Figura 2 - Produção de jogos sérios e seus desafios

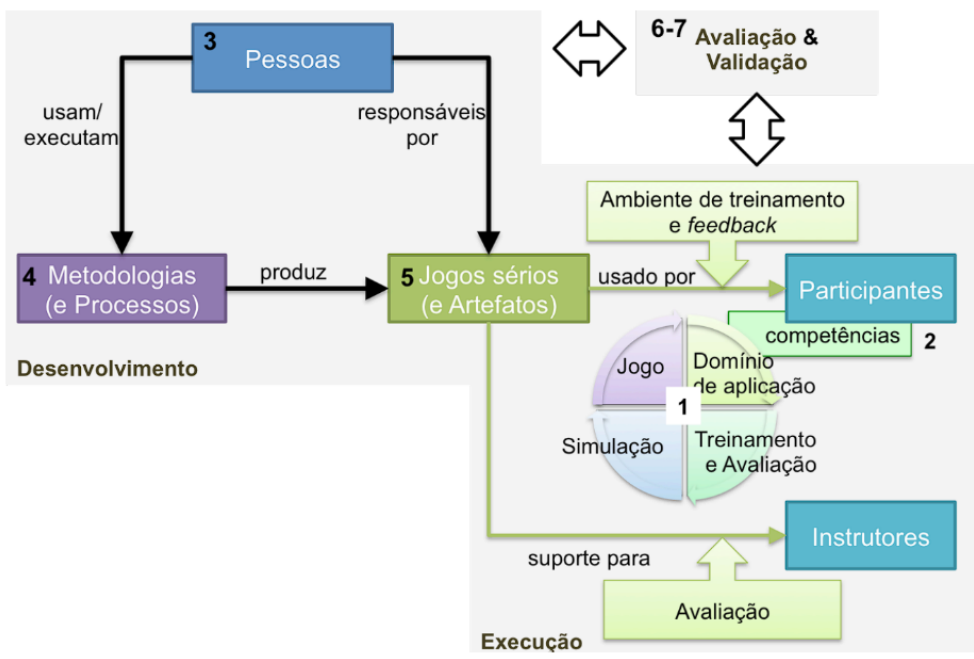

Fonte: Rocha, Bittencourt \& Isotani (2015)

- Características e requisitos do produto: os jogos sérios precisam ter os princípios de aprendizagem e treinamento sem ocorrer a perda de elementos essenciais dos jogos como regras, estratégias, recompensas, feedback contínuo e imediato;

- Treinamento dentro do domínio de aplicação: é necessário conhecer o público-alvo, assim como as competências que serão desenvolvidas e treinadas por meio do jogo. Neste âmbito é importante ressaltar que as competências são formadas por conhecimento, habilidade e atitude pessoal, ou seja, possuir informações, técnica e atitude respectivamente;

- Integração do conhecimento e atividades envolvidas: os envolvidos na elaboração do jogo devem ser dotados de especialistas com domínio de suas respectivas formações;

- Qualidade da metodologia de criação do jogo: este requisito envolve os 4 P's, processos, produtos, projeto e pessoas. Processo (está atrelado a criação dos artefatos); Produto (o próprio artefato); Projeto (controle, planejamento e avaliação durante o processo); e Pessoas (equipe envolvida na criação do artefato).

- Reúso e entendimento do jogo: o jogo precisa ser flexível possibilitando o reúso, a estrutura deve permitir customização, interoperabilidade e integração.

- Integração da avaliação nos diferentes pontos de vistas: durante a elaboração e criação do jogo sério a equipe deve ter de maneira clara o que será avaliado, como será avaliado e quando a avaliação ocorrerá. Este desafio se relaciona com a coleta de avaliações das diferentes pessoas envolvidas.

- Validação: a validação consiste na verificação se os objetivos propostos pelo jogo foram alcançados, uma vez que os jogos sérios têm como propósito ser instrumento de aprendizagem, a validação deve definir o que e como será avaliado o ganho de aprendizado.

\subsection{Desenvolvimento do Jogo}

Seguindo os conceitos expostos, o modelo de engenharia foi analisado como ideal para confecção do jogo. Esse modelo se baseia no desenvolvimento de protótipos que se mantém em constante evolução através dos testes, até atingir os requisitos e objetivos propostos. O modelo tem seu fluxograma representado na Figura 3. 
Figura 3 - Etapas para a confecção do jogo

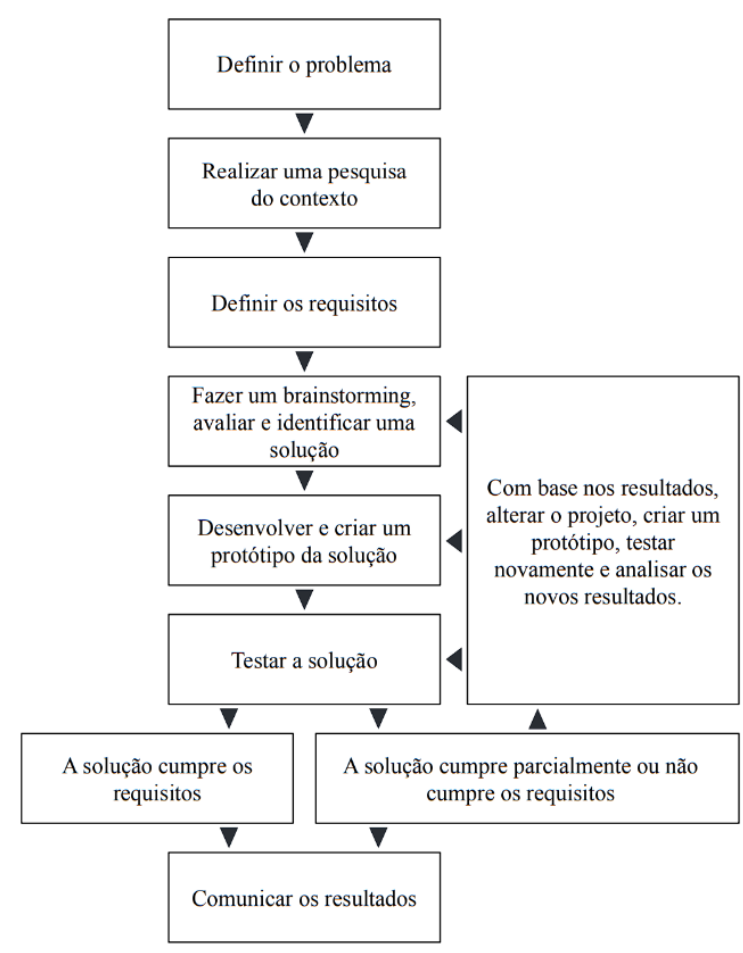

Fonte: Autores (2020)

As duas primeiras etapas foram explicitadas anteriormente com as pesquisas e conceitos que pautaram todo o projeto de desenvolvimento metodológico. Já os requisitos são os sete desafios dos jogos sérios, enquanto se valoriza e aplica os Cores Drives do Octalysis, para gerar um jogo divertido e funcional. Vários estilos de jogos foram elencados e avaliados utilizando além do Octalysis, o método de Forças, Oportunidades, Fraquezas e Ameaças (FOFA). O foco era escolher o jogo mais adaptativo, aquele que pudesse encaixar-se em mais conteúdos, etapas escolares e permitir alterações com base no objetivo e na experiência do aplicador, além de oferecer a possibilidade de ser jogado praticamente sem investimento inicial, levando também em conta a característica mais importante dos jogos, promover a diversão.

Dentre os jogos elencados, o mais adequado foi o RPG. Antes de prosseguir, é importante ter o entendimento do funcionamento e da narrativa do RPG. A sigla se origina do termo em inglês Role Playing Game, que é traduzida como jogo de interpretação de papéis. Comumente é jogado em grupo composto de três a seis pessoas, onde duas partes interagem: o mestre ou também chamado de narrador, que possui a função de contar uma história "aventuresca", e os jogadores, que em contrapartida, escutam-no e interpretam seus personagens, que são participantes do enredo criado pelo narrador, fazendo suas ações diante dos desafios lançados pelo mesmo (FAIRCHILD, 2007).

O RPG é um estilo de jogo marcado pela criatividade, pois sua base é a interpretação de personagens geralmente em um cenário fictício, e cooperação, porque todos se ajudam para superarem as problemáticas narrativas colocadas pelo mestre. A alta flexibilidade e criatividade foi o principal motivo para que se norteasse através do RPG o processo de criação do modelo adequado para o jogo proposto. 
No espectro do RPG, há vários jogos com as mais diversas características, e para o projeto em questão, uma em específica era necessária: que todos os alunos de uma sala pudessem jogar ao mesmo tempo. Isso inviabiliza o professor fazer o papel de narrador, pois não teria como o educador sozinho direcionar a história para todos os estudantes, pois o indicado para um RPG tradicional é de no máximo 5 jogadores para um narrador.

Foi procurado um RPG em que conseguisse flexibilizar a figura do "mestre" e o principal elencado foi o FIASCO, criado por Jason Morningstar. O FIASCO é um jogo focado nas relações entre os personagens e seu maior diferencial é a ausência de alguém fixo como narrador, papel esse que é distribuído entre todos os jogadores (MORNINGSTAR, 2011), ou seja, todos interpretam um personagem e também narram a história. Um fator muito discutido na sua escolha, foi que colocando o próprio aluno como narrador, o projeto também entraria no âmbito das metodologias ativas de ensino, onde o estudante se torna protagonista do seu processo de aprendizado. O FIASCO possui menos Cores Drives do Octalysis, mas isso significa uma simplicidade maior, que é necessária para um jogo que se propõe a ser jogado no período disponível dentro de uma aula.

Após as alterações para que o jogo se adequasse aos objetivos do projeto, foi realizado um teste com a presença de um professor convidado e o mesmo expôs as possíveis dificuldades que o jogo teria para ser aplicado em sala de aula e algumas sugestões para sua melhoria. Seguindo os ajustes sugeridos, foram feitas também outras adequações nas mecânicas para atingir melhor aos sete desafios dos jogos sérios. Assim surgiu o Educa \& Ação, um jogo que objetiva ser adaptável, divertido e focar em desenvolver as habilidades e competências de seus jogadores.

\subsection{Testes do Educa \& Ação}

Com o jogo pronto e seus conjuntos de regras organizados, os testes foram realizados no curso de engenharia civil do IFTO do campus Palmas, nas turmas das matérias de Planejamento e Gerenciamento de Obras, da matriz curricular do $8^{\circ}$ Período, e Gestão da Qualidade e Produtividade, do $10^{\circ}$ Período. O professor responsável pela turma produziu o material para o jogo teste com base no que a equipe do projeto o orientou sobre o jogo e no conteúdo ministrado na matéria. No início do horário da aula, foi apresentado o Educa \& Ação aos alunos, com uma explicação do jogo e como seria a abordagem, após a demonstração a turma foi dividida em grupos de três a quatro acadêmicos, que jogariam entre si, e no decorrer do jogo, os autores ajudavam os grupos que ainda ficaram com dúvidas no percurso da aula.

\section{RESULTADOS E DISCUSSÃO}

Passados os testes, foi coletado o feedback dos alunos primeiramente ainda na sala, para exporem sua primeira opinião e posteriormente por meio de um questionário, para não se sentirem pressionados e poderem desenvolver melhor suas críticas. As trinta respostas coletadas, sendo 14 da turma de Planejamento e Gestão de Obras e 16 da turma de Gestão da Qualidade e Produtividade, foram em relação à possibilidade de aprendizado do Educa \& Ação!, duração do jogo, duração da explicação, clareza da explicação, dificuldade inicial, timidez antes e depois do jogo, experiência geral, se jogaria novamente e, por se tratar de um jogo, diversão ao jogar. Algumas respostas possuem escala de 0 a 10, sendo 0 o pior caso e 10 o melhor. 
Figura 4 - Respostas sobre a duração do jogo

\section{A DURAÇÃO DO JOGO FOI SATISFATÓRIA?}

-Demorado Ideal Rápido

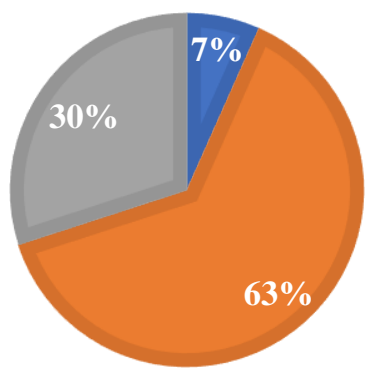

Fonte: Autores (2020)

Figura 5 - Resposta sobre a explicação do jogo

\section{O TEMPO PARA A EXPLICAÇÃO DO JOGO FOI SATISFATÓRIO?}

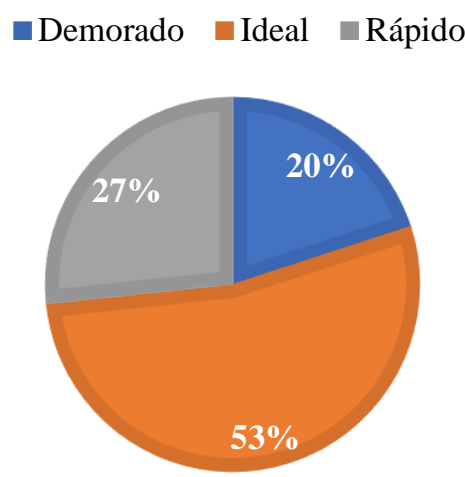

Fonte: Autores (2020)

Em relação aos procedimentos do jogo, conforme Figura 4 e Figura 5, 63,3\% dos alunos consideraram a duração do jogo satisfatória, mostrando que o jogo é aplicável na faculdade. E 53,3\% julgaram o tempo de explicação satisfatório, culminando numa clareza de 6,4 numa escala de 0 a 10. Mesmo no primeiro contato do jogo relacionando um tema tão complexo quanto os abordados, o tempo da aula foi suficiente, mostrando que a limitação de tempo está superada.

Analisando as respostas dos alunos, 96,7\% acreditam que o Educa \& Ação é uma ferramenta que pode contribuir para sua formação, validando o cunho educativo do RPG na engenharia civil até para os alunos no final da graduação. Os 93,3\% dos alunos que jogariam novamente mostram o fator de repetição do jogo, além de validar a possibilidade de jogar em outras matérias.

Outro ponto que o Educa \& Ação se propõe a melhorar é a timidez, que caiu 67,3\%, começando em 5,2 para finalizar em 1,7 numa escala de 0 a 10. Mas a variável mais importante, 
aquela que é o diferencial do Educa \& Ação, é a diversão, e 100\% dos alunos acharam o jogo divertido. Fechando com uma nota de experiência geral dos alunos de 8,2.

O jogo foi apresentado à professora da disciplina de Tecnologias da Educação e coordenadora do subprojeto do Programa Institucional de Bolsa de Iniciação à Docência (PIBID) do curso de Letras do campus Palmas. Como professora experiente na área de metodologias ativas, ela aprovou o Educa \& Ação, além de adaptá-lo como uma atividade do PIBID no $9^{\circ}$ ano de uma escola municipal da cidade.

\section{CONCLUSÃO}

A partir dos resultados obtidos, o Educa \& Ação mostrou-se uma metodologia eficiente, ao usar apenas o tempo de uma aula desde sua explicação até o final do jogo, além de sua adaptabilidade, podendo ser aplicada em várias disciplinas pelo seu grande fator de repetição, perpassando e melhorando a timidez. Também foi reconhecida como eficaz, ao ser avaliada como uma ferramenta que agrega à formação de engenharia civil pelos próprios graduandos.

Os resultados educacionais também foram reconhecidos por profissionais e futuros profissionais da educação, como os docentes e discentes do curso de licenciatura em Letras do Campus Palmas, que inclusive o testaram no ensino fundamental, validando assim a adaptabilidade da metodologia na área de conhecimento, nível escolar e idade do público-alvo.

É possível concluir que o Educa \& Ação é uma metodologia ativa competente para a graduação de engenharia, por mostrar um caminho novo e pautado pelos requisitos dos jogos sérios para o aprendizado, colocando o graduando em uma situação em que ele ensina e aprende ao mesmo tempo com os colegas de sala. $\mathrm{O}$ graduando ganha experiência prática na área, tendo que tomar decisões e lidar com outros profissionais, tudo isso enquanto se diverte em um ambiente controlado e trabalhado pelo professor.

\section{Agradecimentos}

À professora Dra. Mirelle Freitas, por apoiar a aplicação do Educa \& Ação! no PIBID de Letras do campus.

Ao licenciando de Letras Rodrigo Batista, que acreditou e contribuiu do teste do Educa \& Ação e aos demais que o ajudaram na aplicação.

Ao professor Rômulo, que nos ajudou no teste da brainstorm.

\section{REFERÊNCIAS}

CHOU, Yu-kai. Octalysis - the complete Gamification framework. Disponível em: https://yukaichou.com/gamification-examples/octalysis-complete-gamification-framework/. Acesso em: 12 ago. 2019.

FAIRCHILD, Thomas M. Leituras de impressos de RPG no Brasil: o satânico e o secular. 2007. 442 f. Tese (Doutorado) - Faculdade de Educação, Universidade de São Paulo, São Paulo, 2007. 
IEL, Núcleo Central e SENAI. Inova Engenharia: propostas para a modernização da educação em engenharia no Brasil. $1^{a}$ edição, Brasília: IEL.NC/SENAI.DN, 2006. Disponível em: http://www.nece.ctc.puc-rio.br/publicacoes/INOVA_ENGENHARIA.pdf. Acesso em: 10 dez. 2019.

MORNIGSTAR, Jason. Fiasco. $1^{a}$ edição, Curitiba: Retropunk Publicações, 2011. Tradução de: J.M. Trevisan.

QUERINO, R. A.; BORGES, M. L. As ciências humanas e o currículo por competências na engenharia civil: o projeto político-pedagógico da Universidade de Uberaba. In: CONGRESSO BRASILEIRO DE ENSINO DE ENGENHARIA, 30., 2002, Piracicaba. Anais eletrônico. Piracicaba: ABENGE, 2002. Disponivel em:

http://www.abenge.org.br/cobenge/arquivos/17/artigos/6.pdf. Acesso em: 01 mai. 2020.

ROCHA, Rafaela Vilela da; BITTENCOURT, Ig Ibert; ISOTANI, Seiji. Análise, Projeto, Desenvolvimento e Avaliação de Jogos Sérios e Afins: uma revisão de desafios e oportunidades. In: XXVI Simpósio Brasileiro de Informática na Educação, 2015, Maceió. Anais. Maceió, 2015. Disponível em: http://www.brie.org/pub/index.php/sbie/article/download/5342/3705. Acesso em: 12 ago. 2019.

UNESCO. Informe de resultados TERCE: factores asociados; resumen ejecutivo. Disponível em: https://unesdoc.unesco.org/ark:/48223/pf0000243979. Acesso em: 3 fev. 2020.

\title{
EDUCA \& AÇÃO: AN ACTIVE METHODOLOGY FACING THE ENGINEERING COURSE TO SIMULATE REALITY THROUGH THE GAME
}

\begin{abstract}
Placing the student as the protagonist of his own teaching and extrapolating the barriers of the conventional classroom favors his learning (QUERINO \& BORGES, 2002). One way to achieve this is to adopt methodologies active in the process, since positive interactions in classes also contribute to the student's knowledge (UNESCO, 2013). Starting from this point, a methodology was developed through gamification to be used in graduation. For this, the structures of Octalysis, by Chou (2019), and serious games, by Rocha, Bittencourt \& Isotani (2015), were applied to find and adapt the role-playing system that best fits the classroom: FIASCO, by Morningstar (2011). And so Educa \& Ação was developed, a system aimed at working with skills that are often not dealt with in college, while the student has a different, fun and cooperative class. Tests were carried out in the civil engineering course of the IFTO Campus Palmas in the disciplines of Planning and Management of Works, of the curricular matrix of the 8th Period, and Quality and Productivity Management, of the 10th Period. The results obtained by the students' feedbacks were positive: $96.7 \%$ see Educa \& Ação as a tool that contributes to their graduation, $93.3 \%$ would play again and $100 \%$ had fun while playing. With an overall experience score of 8.2, Educa \& Ação is a validated methodology for teaching in civil engineering.
\end{abstract}

Keywords: Teaching, active methodology, RPG, civil engineering. 